\title{
MUfASA: Simulations of Galaxy Formation for MeerKAT Science
}

\section{R. Davé êt, $^{1,3 *}$ M. Rafieferantsoa ${ }^{1,2}$}

${ }^{1}$ University of the Western Cape, Bellville, Cape Town

${ }^{2}$ South African Astronomical Observatory, Observatory, Cape Town

${ }^{3}$ African Institute for Mathematical Sciences, Muizenberg, Cape Town

\begin{abstract}
We present the MUFASA simulations, a new suite of cosmological hydrodynamic simulations for galaxy formation and intergalactic medium evolution, and describe some use cases for MeerKAT science. The MUfASA simulations use state of the art modeling techniques and updated microphysics prescriptions to produce among the most realistic descriptions of the galaxy population ever achieved numerically. Here we outline various avenues by which MUFASA will be used to help guide and interpret observations to be done with MeerKAT, focusing particularly on the LADUMA, MIGHTEE, MHONGOOSE, and MALS Key Project Surveys.
\end{abstract}

MeerKAT Science: On the Pathway to the SKA,

25-27 May, 2016,

Stellenbosch, South Africa

\footnotetext{
*Speaker.

†romeeld@gmail.com
} 


\section{Introduction}

Galaxy formation is a complex problem. The concordance cosmological model with dark matter and dark energy now provides a firm prediction for the gravitationally-driven backbone of large-scale structure within which galaxies grow. However, the baryonic processes associated with the accumulation of gas, the formation of stars and black holes, and the return of mass, energy, and metals back into surrounding gas remain at best only heuristically understood.

Cosmological simulations of galaxy formation have progressed dramatically in the last few years, driven both by a better understanding of the physical processes involved in galaxy formation [1] as well as ever-increasing computational power. Such simulations can now reproduce the basic demographics of the galaxy population, which makes them plausible and viable tools to help understand the growth of galaxies within a fully cosmological context.

Hydrodynamic simulations have, over the past decade, driven a paradigm shift in the basic questions of galaxy formation. Previous analytic-based modeling focused on the connection of galaxies to dark matter, and tied the growth of galaxies to the merger history of their dark matter halos. In the modern view, understanding galaxy formation is regarded as tantamount to constraining the cycle of gas, metals, and energy into and out of galaxies, which has come to be called the baryon cycle.

Detecting gas in and around galaxies is challenging. While stars shine brightly at optical wavelengths, gas is typically more diffuse, hence fainter, and a complete census of all gas phases is only possible by observing a wide range of wavelengths from X-rays to radio. An important gas component that has been up till now infeasible to explore over much of cosmic time is neutral hydrogen. As gas moves from the ionised intergalactic medium (IGM) into the dense interstellar medium (ISM), it must pass through a neutral phase. H I 21-cm data from MeerKAT will be able to quantify the evolution of $\mathrm{H} \mathrm{I}$ in galaxies with existing multi-wavelength data, as well as to probe the far outskirts of the baryon cycle around local galaxies to unprecedented depth. These data will be crucial in firming up a glaring hole in our observational census of gas in the baryon cycle.

Interpreting this data within a multi-wavelength evolutionary context will be the role of galaxy formation models. Since the neutral gas around galaxies represents a dynamical (non-equilibrium) phase, it is crucial to utilise fully dynamical models to properly and robustly model this component. Hydrodynamic simulations have thus begun to examine the predicted $\mathrm{H}$ I content of galaxies as a function of redshift [2], environment [3], and dynamical state [4]. These simulations can at the same time be used to examine the growth of stars, gas (in various phases), and black holes within a $\Lambda \mathrm{CDM}$ framework, thereby providing a coherent context for how multi-wavelength data can trace various aspects of the baryon cycle.

In these proceedings we present the MUFASA simulations, which are a new set of state-ofthe-art cosmological galaxy formation simulations developed and run here in South Africa. We discuss plans to utilise these simulations to interface with observations from four MeerKAT Key Science Projects: LADUMA, MIGHTEE, MHONGOOSE, and MALS. Finally, we discuss plans to interface MUFASA simulations with the CyberSKA project at the Institute for Data Intensive Astronomy (IDIA). 


\section{The Mufasa project}

The MUfASA simulations are fully described in [5]; here we provide a brief summary. The idea of MUFASA is to utilise, whenever possible, the results from high-resolution zoom simulations or analytic models to constrain the most uncertain sub-resolution parameters associated with star formation and feedback, rather than simply tuning parameters to fit data. Nonetheless, MUFASA turns out to also be highly successful at fitting data, thus validating this "multi-scale" approach to building models of galaxy formation.

Key new features of MUFASA include:

- The use of a new "meshless" hydrodynamics Gizmo code [6] for solving the equations of hydrodynamics that marries the advantages of particle- and mesh-based approaches, thereby improving the accuracy over both.

- A molecular gas-based star formation law employing the analytic formalism based on theoretical ISM models [7], along with minimal ISM pressurization to keep the Jeans mass fully resolved.

- Scalings for outflow mass loading and velocity taken from the Feedback In Realistic Environments self-consistent zoom simulations [8].

- Quenching in massive galaxies based on keeping hot gas hot [9], using an evolving quenching halo mass scale based on the analytic equilibrium model constraints from [10].

- Explicit two-phase winds, with hydrodynamic decoupling in order to enable "chimney-style" escape from the ISM.

- 9-metal chemical evolution with contributions from supernovae Type II and Type Ia, and asymptotic giant branch stars.

Remarkably, these physically-motivated prescriptions result in some of the most accurate agreement between predictions and observations for key basic galaxy demographics ever seen in galaxy formation models. There are still significant discrepancies particularly at intermediate redshifts, but here the observational uncertainties are larger. [5] showed that the agreement in the benchmark galaxy stellar mass function was excellent over most of cosmic time. [11] extended this to show good agreement in the gas (molecular and atomic), metal, and star formation rate properties of galaxies. We note that other recent simulations such as EAGLE [12] achieve similar levels of success. Nonetheless, these successes highlight MUFASA as a leading platform for examining the physical processes of galaxy formation and their observational consequences that might be traced using multi-wavelength observations. Here, we will focus on the observational predictions in the radio regime.

\section{MeerKAT science with MUfASA}

As mentioned, MUfASA has the potential to interface with a wide range of extragalactic science cases. Some science, however, requires new aspects to be developed for MUFASA, which are 
in process. Here we describe the basic concepts and mechanisms for how MUFASA will interface with MeerKAT Key Science Projects.

\subsection{LADUMA}

LADUMA is described more fully in the proceedings from Blyth, but in brief it is a $3000+$ hour pointing on a single square degree in the Extended Chandra Deep Field South. The key science goal is to study the evolution of neutral hydrogen in galaxies, in particular the H I mass function (HIMF), out to $z \approx 1.4$. The extensive multi-wavelength data in the ECDFS will provide ample opportunities to relate $\mathrm{H}$ I content with many other physical and environmental properties of galaxies, providing a new test of galaxy formation models.

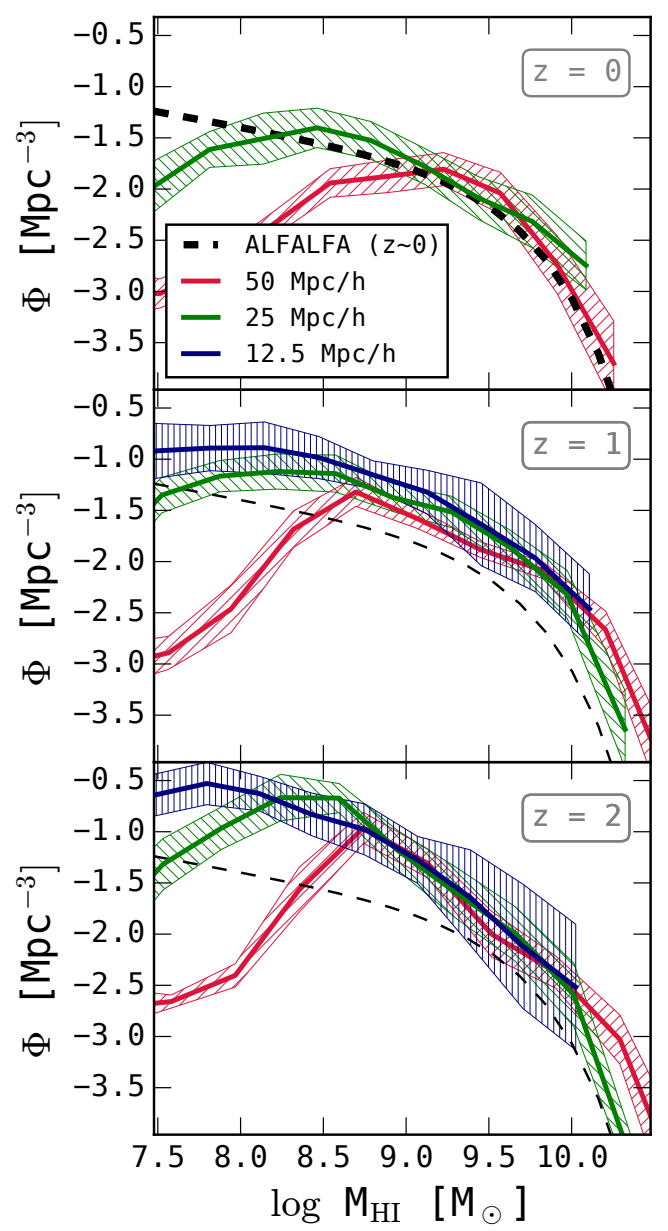

Figure 1: H I mass functions in the $50 h^{-1} \mathrm{Mpc}$ (red solid), $25 h^{-1} \mathrm{Mpc}$ (green dashed), and $12.5 h^{-1} \mathrm{Mpc}$ (blue dotted, for $z \geq 1$ ) MUFASA simulations. Hatched region shows the cosmic variance computed over 8 sub-octants in each volume. Black dashed line shows $z \approx 0$ observations from the ALFALFA survey, reproduced at $z=1,2$ in order to better depict the evolution. MUFASA does reasonably well reproducing the HIMF over the $M_{H I}$ range of convergence; at $z \sim 0$, the $50 h^{-1} \mathrm{Mpc}$ box begins to deviate from the $25 h^{-1} \mathrm{Mpc}$ at $M_{H I}<\sim 10^{9} M_{\odot}$, while at higher redshifts this occurs at $M_{H I} \sim 10^{8.7} M_{\odot}$. The HIMF generally increases in amplitude to higher redshifts, and also steepens noticeably at $z=2$. There is fair resolution convergence down to $M_{H I} \sim 10^{9} M_{\odot}$ in the $50 h^{-1} \mathrm{Mpc}$ volume, and to $\approx 8 \times$ lower in the $25 h^{-1} \mathrm{Mpc}$ volume. 
Figure 1, taken from [11], shows the predictions for the evolution of the HIMF from MUFASA. Encouragingly, these predictions turn out to be somewhat more optimistic at $z \sim 1$ than our previous simulations in [2] in terms of the number of galaxies expected. Basically, the MUFASA HIMF mass function increases in amplitude (i.e. number density) by about a factor of 3 from $z=0 \rightarrow 1$. This would result in significantly more massive $\mathrm{H}$ I galaxies observed at $z \sim 1$ than originally expected from a non-evolving HIMF.

More interesting than the HIMF will be the ability to connect H I to other multi-wavelength galaxy properties such as stellar mass, molecular gas mass, star formation rate, metallicity, and environment. With complete ancillary spectroscopy over the 1 square degree of ECDFS, this will enable detailed studies of scaling relations versus $\mathrm{H}$, which provides crucial tests of models and insights into the physics governing galaxy growth.

All of these properties are straightforwardly predictable in MUFASA, and in large part have already been done. The main work left is to develop more realistic methods to mock the observations, through light cones and adding instrumental noise characteristics.

\subsection{MIGHTEE}

MIGHTEE is described in the contribution from Taylor and Jarvis. It aims to cover $\sim 20$ square degrees in the VIDEO fields, effectively providing a wide-area wedding cake counterpart to LADUMA. Its primary focus is continuum science, particularly AGN and star formation activity, but will commensurately obtain $\mathrm{H}$ I data to better probe the massive end of the HIMF.

The currently published MUFASA runs do not include black hole growth. However, this has now been implemented into GIZMO-MUFASA, following the novel torque-limited accretion model, including BAL winds for feedback [13]. We are busy recalibrating this model with various other minor improvements in order to run the next set of cosmological simulations, including self-consistent black hole growth and feedback.

With this, we will directly predict the population of black holes in our simulated Universe, along with their accretion rates. With canonical assumptions, it is then possible to turn this into an AGN luminosity function, particularly focusing on the Seyfert galaxy regime that will be exhaustively probed by MIGHTEE out to $z \sim 2-3$. A crucial question is to understand how closely stellar and black hole growth correlate, because this provides a key discriminant between current models of black hole growth such as torque-limited versus Bondi accretion. With MIGHTEE, it should be possible to directly trace the growth of the majority of black hole mass in the Universe, which will be a valuable new constraint on models of black hole-galaxy co-evolution.

Another important aspect of MIGHTEE is to examine extinction-free SFRs, particularly at intermediate redshifts. It has long been a generic issue with cosmological galaxy formation models that they underpredict the SFR by $\sim \times 2-3$ at a given stellar mass around $z \sim 2[14,1]$. Except at the highest masses, the observed SFRs typically come from UV-based or line emission measures with large extinction corrections. Using the radio as a direct tracer of the Type II supernova rate to obtain SFRs provides a valuable independent check of such data, and will be crucial for resolving this key outstanding issue in current galaxy formation theory.

Finally, MIGHTEE will be particularly revolutionary for studying cosmic magnetic fields. Recent results suggest that magnetic fields are more pervasive than previously believed in the IGM [?]. 
Using Mufas A, we are working with R. Taylor and L. Hunt to understand how the distribution of cosmic magnetic fields can be tested and constrained with MIGHTEE data.

\subsection{MHONGOOSE}

MHONGOOSE is an H I 21-cm survey of 30 nearby galaxies spanning a wide range of properties, particularly targeting the outskirts down to very low column densities below $10^{17} \mathrm{~cm}^{-2}$. The goal is to understand the distribution and morphology of $\mathrm{H} \mathrm{I}$ as it connects galaxy outskirts to their surrounding circum-galactic medium (CGM).

With $\sim$ kpc-scale resolution, MUfASA is well situated to study how H I relates to the CGM. Currently, the CGM of external galaxies can only be probed via absorption line spectroscopy, typically in the UV where the H I Lyman series and many metal lines are found. This is limited to finding galaxies with nearby (in projection) bright background sources, which is rare, and additionally only gives 1-D information. Our group is among the leaders in CGM studies in simulations, being part of the COS-Halos team [16]. We are now gearing up for similar absorption line studies in MUFASA.

Ideally, one would like to image the CGM directly. However, this is difficult in H I because current surveys cannot even reach $10^{18} \mathrm{~cm}^{-2}$ in column density, leaving a gap in column density coverage between $21-\mathrm{cm}$ emission and absorption that is precisely in the crucial range where accreting gas becomes self-shielded and hence neutral. MHONGOOSE will fill this gap, and connect such studies to provide a more comprehensive view of how galaxies connect to the CGM.

While MufASA's resolution is good, ideally one would like to resolve the detailed structure of $\mathrm{HI}$ in and around galaxies, which requires more like $\sim 100 \mathrm{pc}$ resolution. For this, we are now running "zoom" simulations, where we extract galaxies from the MUFASA cosmological run and re-simulate their individual halos at much higher resolution, while retaining the large-scale context at lower resolution. This will enable us to achieve the required resolution with feasible computational cost.

The next step is to turn MUFASA outputs into 21-cm data cubes. We are working with several groups in order to do this, including adding instrumental effects. The key in simulations is that we have the dynamical history of all the gas, which allows us to directly relate the predicted $21-\mathrm{cm}$ emission with inflowing, outflowing, or ambient gas [17]. By constructing maps made from these individual components, we can directly assess how $\mathrm{HI}$ emission is probing the baryon cycle of inflows and outflows in the CGM around galaxies [4], and thus provide crucial interpretation of MHONGOOSE data towards this central question of galaxy formation.

\subsection{MALS}

MALS is a $21-\mathrm{cm}$ absorption line survey of $\sim 1000$ bright radio sources, to look for intervening $\mathrm{H}$ I galaxies at unprecedented sensitivities. The goal is to directly measure the evolution of cosmic $\mathrm{H}$ I and (sub-)damped Lyman alpha (DLA) systems at $0<z<1.5$, with extremely high $\sim 5 \mathrm{~km} / \mathrm{s}$ spectral resolution. It is difficult to probe this regime for DLAs in the canonical UV band since it requires space-based spectroscopy, so MALS will make important contributions.

Our group has long been one of the leaders in studying absorption line probes of the cosmos [15]. It is relatively straightforward to make spectra for 21-cm rather than UV absorption 
lines. The primary limitation will be that the cosmic volume covered by MALS will be far larger than our simulation volume, so we must push to larger volumes, which requires more fluid elements and hence more comptutational resources. Nonetheless, even with existing MUFASA simulations we can already make substantial progress.

Key questions include: What is the cosmic evolution of neutral gas, and does it agree with the MUFASA predictions of [11]? What is the nature of these DLA absorbers, and does the nature change as a function of column density? How do these DLAs relate to the underlying galaxy population? Can the dynamics of $\mathrm{H}$ I absorption tell us about feedback processes? These questions and more we can address with MUFASA and its successors.

\subsection{IDIA}

Numerical theory requires four main stages: Development, running, analysis, and dissemination. The previous sections demonstrate our current capabilities and plans in the first three areas. Dissemination, however, has often lagged behind. Our goal is to use the IDIA platform to provide a comprehensive dissemination portal for MUFASA simulation outputs and catalogs. Physical, photometric, and environmental information will be provided. We have developed easy-to-use python based analysis tools that can be run on the fly to generate catalogs on demand. Eventually, this will include "mock surveys" made from light cones and with all instrumental characteristics included, that can be compared side-by-side with observations.

\section{Conclusion}

The Mufas a simulations represent a state of the art platform for studying multi-wavelength galaxy formation, and hence are an ideal tool for guiding and interpreting extragalactic radio observations with MeerKAT. These simulations, and its successors that include more and better physics, will be utilised for at least four MeerKAT Key Science Projects, namely LADUMA, MIGHTEE, MHONGOOSE, and MALS. Such simulations provide a way to comprehensively situate all these observations, along with ancillary multi-wavelength data, within a current cosmological large-scale structure context. The key goal is to understand how to use these observations to study the baryon cycle of gas flows that are believed to regulate galaxy and black hole growth. By combining MUFASA with MeerKAT observations of the neutral hydrogen content, stellar and black hole growth, and absorption line kinematics across an unprecedented range of regimes and cosmic epochs, we aim to develop a more complete scenario for how galaxies grow and evolve in concert with their surrounding cosmic ecosystem.

\section{References}

[1] Somerville, R. S., Davé, R. 2015, ARA\&A, 53, 51

[2] Davé, R., Katz, N., Oppenheimer, B. D., Kollmeier, J. A., W einberg, D. H. 2013, MNRAS, 434, 2645

[3] Rafieferantsoa, M., Davé, R., Anglés-Alcàzar, D., Katz, N., Kollmeier, J. A., Oppenheimer, B. D. 2015, MNRAS, 453, 3980

[4] Crain, R. A. et al. 2017, MNRAS, 464, 4204 
[5] Davé, R., Thompson, R. J., Hopkins, P. F. 2016, MNRAS, accepted

[6] Hopkins, P.F. 2015, MNRAS, 450, 53

[7] Krumholz, M. R., McKee, C. F., Tumlinson, J. T. 2008, ApJ, 693, 216

[8] Muratov, A.L., Kereš, D., Faucher-Giguere, C. A., Hopkins, P. F., Quataert, E., Murray, N. 2015, MNRAS, 454, 2691

[9] Gabor, J. M. \& Davé, R. 2015, MNRAS, 447, 374

[10] Mitra, S., Davé, R., Finlator, K. 2015, MNRAS, 452, 1184

[11] Davé, R., Rafieferantsoa, M., Thompson, R. J., Hopkins, P. F. 2017, MNRAS, submitted

[12] Schaye, J. et al. 2015, MNRAS, 446, 521

[13] Anglés-Alcázar, D., Davé, R., Faucher-Giguere, C.A., Özel, F., Hopkins, P.F. 2016, MNRAS, submitted, arXiv:1603.08007

[14] Davé, R. 2008, MNRAS, 385, 147

[15] Davé, R., Oppenheimer, B.D., Katz, N., Kollmeier, J.A., Weinberg, D.H. 2010, MNRAS, 408, 2051

[16] Ford, A.B., Werk, J.K., Davé, R. et al. 2016, MNRAS, 459, 1745

[17] Ford, A.B., Davé, R., Oppenheimer, B.D. et al. 2014, MNRAS, 444, 1260

[18] Kim, K. S., Lilly, S. J., Miniati, F., Bernet, M. L., Beck, R., O’Sullivan, S. P., Gaensler, B. M. 2016, ApJ, 829, 133 